\title{
Fabrication and characterization of metallic nanowires
}

\author{
C. Untiedt, G. Rubio, S. Vieira, and N. Agrait \\ Instituto Universitario de Ciencia de Materiales "Nicolás Cabrera,"' Laboratorio de Bajas Temperaturas, \\ Departamento de Física de la Materia Condensada, C-III, Universidad Autónoma de Madrid, 28049 Madrid, Spain
}

(Received 19 December 1996)

\begin{abstract}
The shape of metallic constrictions of nanoscopic dimensions (necks) formed using a scanning tunneling microscope is shown to depend on the fabrication procedure. Submitting the neck to repeated plastic deformation cycles makes it possible to obtain long necks or nanowires. Point-contact spectroscopy results show that these long necks are quite crystalline, indicating that the repeated cycles of plastic deformation act as a "mechanical annealing" of the neck. [S0163-1829(97)05227-2]
\end{abstract}

\section{INTRODUCTION}

The development of new experimental techniques such as mechanically controllable break junctions (MCBJ's) and the scanning tunneling microscope (STM) has made possible the formation and study of atomic-size junctions or contacts between macroscopic metals. Both techniques allow for a very precise control of the relative position of two electrodes which is the key to the study of these nanojunctions. These related techniques differ in the way the nanojunction is formed: in the case of the STM the tip is driven into the substrate to form a large contact and then it is pulled back, while in the MCBJ a fine wire is pulled until it fractures and the two fragments are brought back into contact.

Superconducting and normal contacts have been extensively studied at liquid-helium temperature (4.2 K) (Ref. 1-5) and normal contacts at room temperature. ${ }^{6,7}$ More recently the mechanical properties and their correlation with the conductance have been studied using a combination of force and tunneling microscopy techniques. ${ }^{8-10}$

Theoretically, atomistic mechanisms during plastic deformation of the contact ${ }^{11-13}$ and their effect on the conductance $^{14}$ have been investigated using moleculardynamics (MD) simulations and a tight-binding approximation, and different geometries have been considered by conductance calculations using a free-electron approximation. ${ }^{15-18}$

In spite of all this effort, no direct experimental geometrical and structural information of these necks has been presented. In this paper we present a simple model that makes it possible to obtain an estimate of the shape of the necks and their evolution during plastic deformation. We will also show that the type of neck produced depends on its deformation history and that it is possible to fabricate long necks or nanowires. These nanowires are quite crystalline, as shown by their electronic transport properties at low temperatures.

\section{DETERMINATION OF THE GEOMETRY OF NECKS}

In STM and MCBJ experiments on nanojunctions, the current that traverses the contact at a small constant bias voltage (several milivolts) is measured as a function of the relative displacement of the electrodes. Before a metallic contact is established, the electronic current is due to the tunneling effect and the dependence of the current with distance is exponential. ${ }^{19,3}$ Metallic contact is signaled by an abrupt increase of the current (the conductance for this first contact is of the order of $2 e^{2} / h$, where $e$ is the electron charge, and $h$ is Planck's constant). The variation of the current with the relative displacement of the electrodes depends on how the junction deforms plastically, and can be quite diverse, as attested by Fig. 1, where we show four different sets of STM experimental curves.

In this section we will first discuss the conductance of an ideal metallic constriction and its relation to the minimal cross-sectional area, and then we will present a simple slab model for the evolution of the constriction with relative displacement which makes it possible to obtain an estimate of the shape of the constriction and mechanical properties of the neck.

\section{A. Conductance of a metallic constriction}

Electronic transport through a metallic constriction whose dimensions are smaller than the mean free path of the

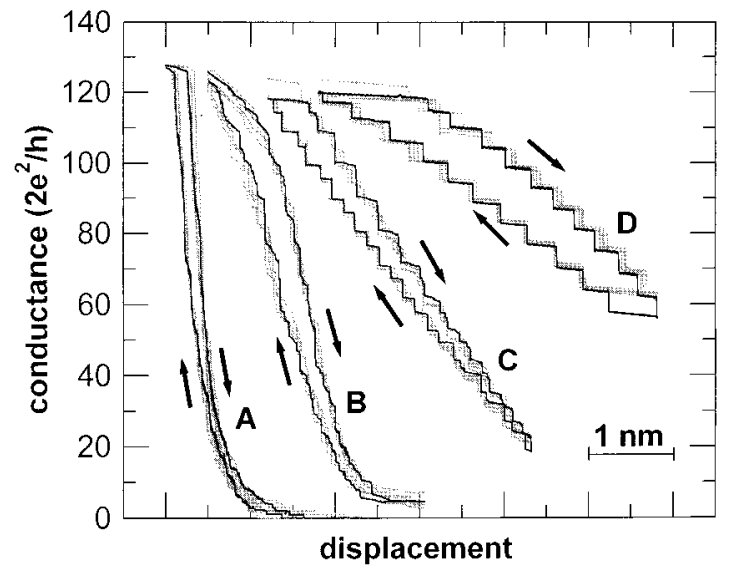

FIG. 1. Four different sets of experimental conductance vs relative tip-substrate displacement cycles for $\mathrm{Au}$ at $4.2 \mathrm{~K}$. Each set consists of five consecutive elongation-contraction cycles (one of the cycles is colored black, while the other four are gray for clarity). The arrows indicate the direction of motion. 
electrons ${ }^{20}$ is ballistic. The conductance of such a constriction was first derived semiclassically by Sharvin. ${ }^{21}$ More recently Torres, Pascual, and Sáenz, ${ }^{16}$ using an exact quantum calculation, derived a corrected version of the Sharvin formula,

$$
G_{\infty} \approx \frac{2 e^{2}}{h}\left(\pi \frac{A}{\lambda_{F}^{2}}-b \frac{P}{\lambda_{F}}\right),
$$

where $\lambda_{F}$ is the Fermi wavelength; $A$ and $P$ are the area and perimeter of the minimal cross section; and $b$ is a parameter that depends on the shape of the neck, being $\frac{1}{2}$ for a cylindrical tube $(\theta=0)$ and $\frac{1}{4}$ for a circular hole $(\theta=\pi / 2)$. The classical Sharvin prediction $^{21,22}$ corresponds to $b=0$. As the contact area increases, the correction due to the perimeter becomes relatively less important (about 5\% for a contact radius of $2 \mathrm{~nm}$ for $\mathrm{Au}$ ). A more realistic approximation to a real constriction that takes into account the work function of the material instead of a hard wall potential ${ }^{23}$ reduces this correction even further.

Equation (1) can be used to estimate the minimal cross section of the constriction. Note that if the neck is ballistic and not too small, the error introduced by not knowing the aperture angle is negligible. However, the existence of defects or disorder would have the effect of decreasing the conductance for a given geometry, and using Eq. (1) would underestimate the neck diameter. These effects were studied by Todorov and co-workers using a molecular-dynamics simulation to obtain the evolution of the atomic positions during plastic deformation of the neck, and a tight-binding model for the conductance corresponding to a given atomic configuration. ${ }^{14}$ These authors found a reduction in the conductance of up to $20 \%$ with respect to the free-electron result. Rough boundaries ${ }^{18}$ also affect the oscillating structure due to the opening and closing of conductance channels, but the smooth part of the conductance is still well described by the modified Sharvin formula. More extended defects would cause an even larger reduction. ${ }^{24}$

Experimental information on the degree of disorder in the constriction can be obtained using point-contact spectroscopy. ${ }^{22}$ The derivative of the differential conductance of a point contact (PC) contains information about the inelastic electron backscattering. For ballistic point contacts the dominant inelastic scattering mechanism is phonon scattering, and the Eliashberg function for the electron-phonon interaction in the point-contact situation, or point-contact spectroscopic (PCS) curve, is given by ${ }^{25}$

$$
\alpha^{2} F_{p}=-\frac{3}{32 \sqrt{2}} \frac{h^{3 / 2} k_{F}^{2}}{4 \pi^{2} m} R^{3 / 2} \frac{\mathrm{d}^{2} I}{\mathrm{~d} V^{2}},
$$

where $R=\mathrm{d} V / \mathrm{d} I$ is the differential resistance, $k_{F}$ is the Fermi wave number, and $m$ is the electron mass. The amplitude of the phonon-induced peaks is reduced if there is elastic scattering (for example, due to impurities or defects) in the contact region. Consequently, a large PCS amplitude indicates that the constriction is indeed ballistic.

\section{B. Slab model}

A simple slab model, similar to that used in Ref. 26 can be used to relate the changes in the minimal cross section as

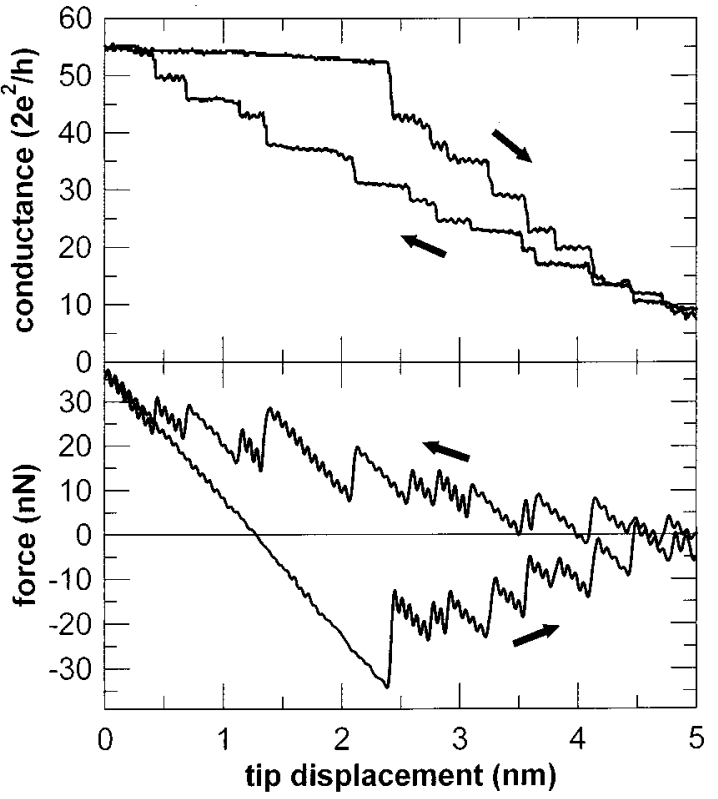

FIG. 2. Simultaneous measurement of conductance and force during an elongation-contraction cycle, for $\mathrm{Au}$ at $300 \mathrm{~K}$. The arrows indicate the direction of motion.

the neck length is varied to the shape of the neck. This model is suggested by the results of combined STM-AFM experiments. ${ }^{10}$ In these experiments, in which the conductance and force as a function of relative displacement are measured simultaneously, it is observed (see Fig. 2) that, when the conductance is rather constant, the force varies linearly, while the abrupt jumps in conductance are correlated to abrupt force relaxations. Between the relaxations deformation is elastic (no energy is dissipated). This correlation has been clearly demonstrated in nanometer-sized contacts (several hundreds of conductance quantum units), ${ }^{8}$ and in atomic-sized contacts (just a few quantum units). ${ }^{10}$ Note that changes in conductance due to effects like conductance quantization would never show as abrupt jumps, but rather as smooth structure in the steps. ${ }^{26}$ This behavior has a clear interpretation: the neck deforms elastically until the stress reaches a critical value (about an order of magnitude larger than the bulk value ${ }^{8,10}$ ), and then relaxes abruptly to a new configuration.

Let us model the neck as a constriction with cylindrical geometry, consisting of slabs of different radii and thicknesses, symmetrical with respect to a plane normal to its narrowest section, as depicted in Fig. 3(a). The elastic properties of the neck, e.g., the Young's modulus $E$ and Poisson's ratio $\mu$ are identical to the bulk values (this is reasonable from a theoretical viewpoint and has also been shown experimentally in Refs. 8 and 10). The basic assumption for this model is that only the narrowest part of the neck deforms plastically. This is likely to be the case because the weakest spot will become the narrowest cross section even if this was not initially the case (due, for example, to the existence of a very defective spot). This assumption could break down for temperatures larger than about $50 \%$ of the melting temperature, for which diffusion will be important.

When the neck is being pulled the central slab will change to a longer (and narrower due to volume conservation) con- 


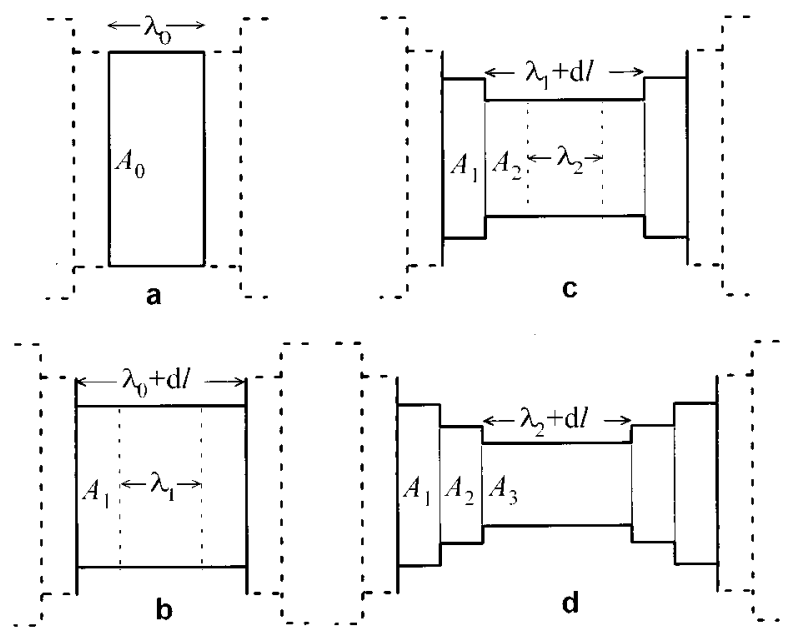

FIG. 3. Plastic deformation of a neck consisting of cylindrical slabs. From one configuration to the next only the central slab elongates, while the rest of the neck does not change.

figuration in order to relax the stress. For instance, if the central slab initially has a cross section $A_{0}=\pi R_{0}^{2}$, and a portion $\lambda_{0}$ of its length is involved in the change of configuration, the new cross section would be $A_{1}=A_{0} \lambda_{0} /\left(\lambda_{0}+\Delta l\right)$, where $\Delta l$ is the change in length [see Fig. 3(b)], and the number of slabs increases by two. After stretching $n$ intervals (assuming the changes in length are always equal to $\Delta l$ ), the increase in length would be $n \Delta l$, the minimal cross section

$$
A_{n}=\frac{A_{n-1} \lambda_{n-1}}{\lambda_{n-1}+\Delta l},
$$

and the spring constant $k_{n}$ of the whole neck

$$
\frac{1}{k_{n}}=\frac{1}{E}\left(\sum_{i=1}^{n-1} \frac{\lambda_{i-1}+\Delta l-\lambda_{i}}{A_{i}}+\frac{\lambda_{n-1}+\Delta l}{A_{n}}\right)+\frac{1}{k_{0}},
$$

where $k_{0}$ is the spring constant of the neck before starting the elongation. The central portion of the neck, consists of slabs of cross section $A_{i}=A_{i-1} \lambda_{i-1} /\left(\lambda_{i-1}+\Delta l\right)$, and thickness $\left(\lambda_{i-1}+\Delta l-\lambda_{i}\right) / 2$. Note that we only know the shape of the pulled portion of the neck but the initial neck is unknown. This parameter $\lambda$, which we may call the plastic deformation length, is related to portion of the neck that changes plastically, and in general it will depend on the cross section, length, and history of the neck. In the limit $\Delta l \rightarrow 0$, the plastic deformation length can be written as $\lambda=-(d \ln A / d l)^{-1}$. Note that $\lambda$ is a phenomenological parameter that will be determine from the experimental data using Eq. (3). We will see that the plastic deformation length varies during plastic deformation of a given neck, and also depends on the fabrication procedure.

As an illustration of how the plastic deformation length reflects on the conductance curves, let us consider the fact that the plastic deformation length at each step depends only the cross section $\lambda=\alpha_{0} A^{n}$, the differential equation for $A$ can be solved analytically. For an initial elongation zero, we have

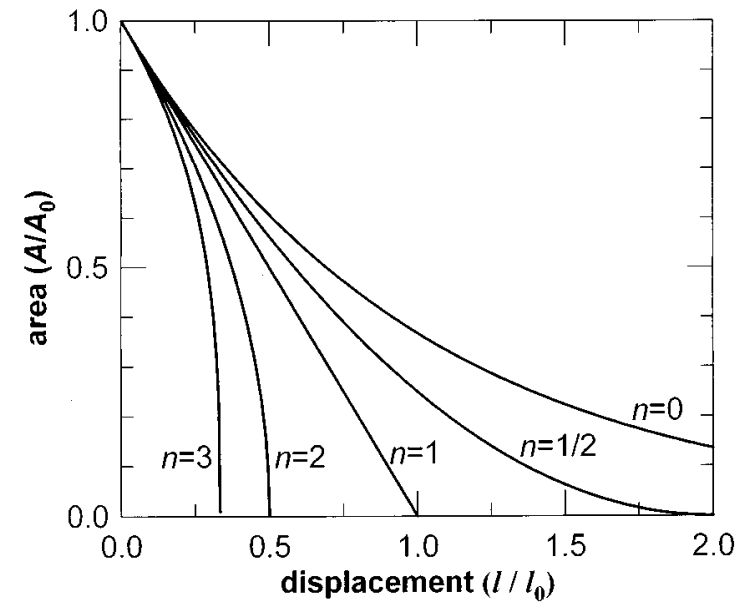

FIG. 4. Evolution of the conductance for different power law dependences of the plastic deformation length $\lambda$ as given by Eqs. (5) and (6).

$$
\begin{gathered}
A=\left[A_{0}^{n}-\frac{\ln }{\alpha_{0}}\right]^{1 / n} \quad \text { for } n \neq 0, \\
A=A_{0} \exp \left(-l / \lambda_{0}\right) \quad \text { for } n=0,
\end{gathered}
$$

where $l$ is the elongation.

The evolution of the minimal cross section, and consequently of the conductance, with the changes in length, is shown in Fig. 4. In Ref. 9, the authors ${ }^{9}$ considered only the case $n=0$. Figure 5 shows a simulation of the neck evolution including the effect of elastic deformation. The black dots represent the equilibrium (relaxed) configurations, and we have assumed that the relaxations occur at a fixed value of

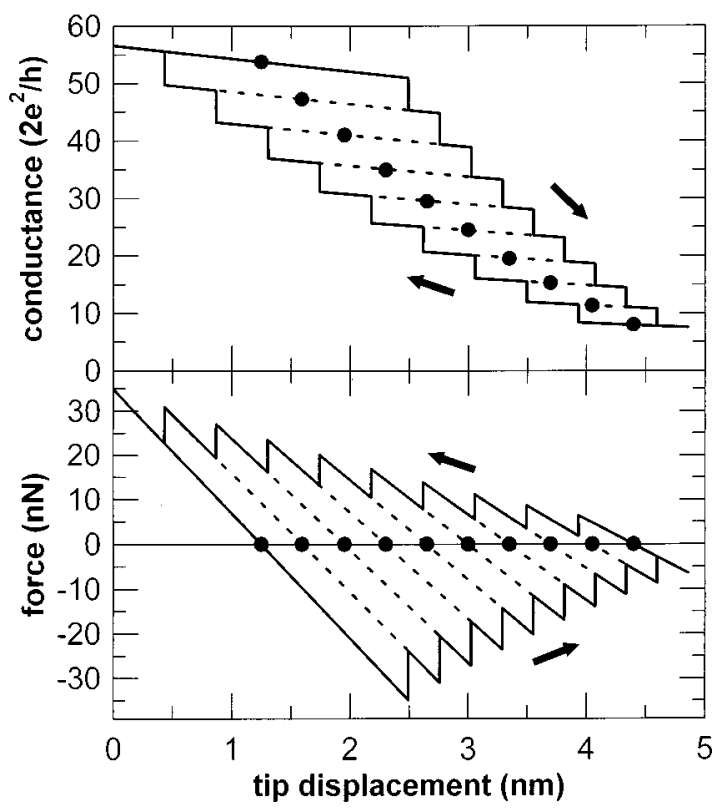

FIG. 5. Simulation using the slab model of the conductance and force during an elongation-contraction cycle. The relaxed position for each configuration is marked with a black dot. We are depicting a fairly ideal case in which the configurations during elongation are the same as the configurations during contraction. 
the apparent pressure (force divided by minimal crosssection area) as observed experimentally. ${ }^{8,10}$

\section{EXPERIMENTAL RESULTS}

\section{A. Fabrication of the necks}

The necks studied in this paper have been obtained using an STM and an STM-AFM combination, and the experiments were performed in different conditions: low temperatures $(4.2 \mathrm{~K})$ in $\mathrm{He}$ exchange gas, room temperature in $\mathrm{He}$ gas, and room temperature and ambient conditions. The fabrication procedure described in this section works with ductile metals like $\mathrm{Pb}, \mathrm{Au}, \mathrm{Al}$, and $\mathrm{Sn}$. In this paper we will present only the results for Au.

The basic procedure consists of crashing a clean metallic tip into a clean metallic substrate in a controlled manner. The STM is convenient because, in addition to the high degree of control on tip-substrate separation (vertical positioning), it makes possible the selection of different spots on the substrate (horizontal positioning). We normally use the same metal for both tip and sample, because this guarantees that the composition of the neck is homogeneous. As the tip is pressed against the substrate, both electrodes deform plastically and then bind by cohesive forces, forming a metal contact. Retraction of the tip results in the formation of a connective neck that elongates plastically and eventually breaks. In all the ductile metals studied to date, rupture takes place through a gradual decrease of the cross section of the neck. Measuring the current through the neck at a fixed bias voltage of 10-100 $\mathrm{mV}$ applied between tip and substrate makes it possible to know the conductance as a function of the displacement of the tip relative to the substrate, and to follow the evolution of the neck. As will be shown, this evolution depends on the history of the contact.

In order to form a metal contact, tip and substrate surface must be clean. Nevertheless, the preparation procedure is not very critical: it is necessary to get rid of oxides and organic contaminants. For $\mathrm{Au}$ samples, sonication in acetone or cleaning in a 3:1 concentrated $\mathrm{H}_{2} \mathrm{SO}_{4}: 30 \% \mathrm{H}_{2} \mathrm{O}_{2}$ solution work fine. For $\mathrm{Pb}$ or $\mathrm{Al}$, scratching the surfaces with a clean blade is adequate. Tips are always clipped, and care must be taken not to produce a very fine tip that could bend elastically, since we want all the deformation (both plastic and elastic) to take place at the contact. It must be emphasized that the actual cleaning of the contacting surfaces takes place during the experiment: the tip is crashed forcibly and repeatedly into the substrate, causing extended plastic deformation until a good metallic contact is obtained. Pressing tip and substrate hard has the effect not only of displacing adsorbates or oxide on the surface but also of involving large amounts of material in the formation process which makes possible the fabrication of longer necks. This fabrication procedure contrasts with the gentle contact formation by other authors both in conductance ${ }^{6,7}$ and force ${ }^{9}$ measurements.

In STM experiments, formation of a good metallic contact is easily recognized because after rupture the apparent tunneling barrier attains a high value $(3-4 \mathrm{eV})$, and separation of the tip and substrate results in the formation of a protrusion on the substrate that can be imaged. In this case the last contact has been shown to be of the order of $2 e^{2} / h$. Formation of a good metallic contact is even easier to recognize in

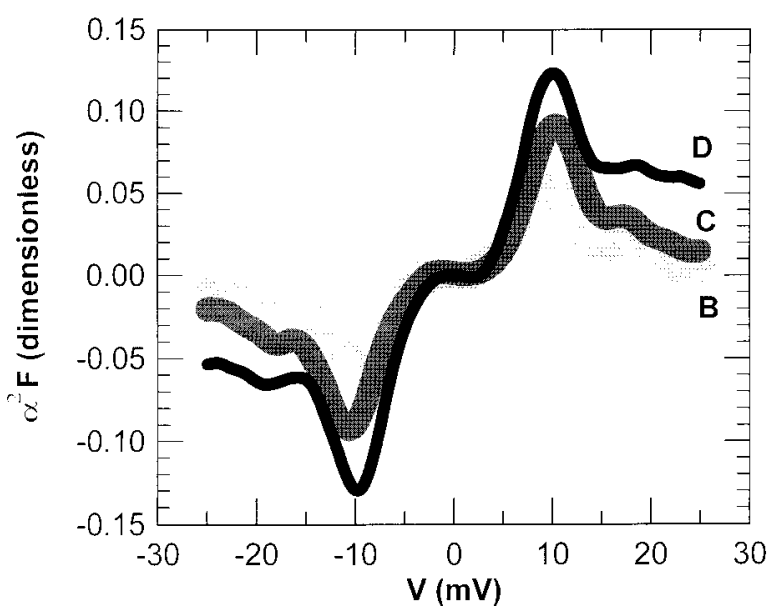

FIG. 6. PCS curves at $4.2 \mathrm{~K}$ for Au necks whose behaviors are similar to those in Fig. 1. The black curve corresponds to a cycle of type $D$, at a conductance of 83 units; dark gray curve corresponds to type $C$, at 77,106 , and 130 conductance units; the light gray curve corresponds to type $B$ at 77, 103, 130 conductance units. All the curves for a given cycle are included within thickness of the plotted curves.

the STM-AFM experiments, since contamination produces a repulsive force while in the tunneling regime.

After the initial preparation of the contact described above, repeated cycles of elongation and contraction with a given amplitude typically result in a very similar current (and force, in the STM-AFM experiments) evolution, that is, a kind of steady state is reached. We must remark that the system remains in this steady state until the amplitude or offset of the cycles is altered, and in some cases as we will see below subsequent cycles are very repetitive. In a marked contrast with macroscopic plastic deformation the contact shows no sign of fatigue. We only present results for these steady-state cycles. The usual data acquisition parameters are 2048 data points per cycle at a rate of $10-100 \mu \mathrm{s}$, and typically sets of five cycles are acquired.

It is important to note that the necks (slope and hysteresis of the cycles) depend strongly on the fabrication procedure. Gentle contact causes necks of type $A$ (see Fig. 1) while repeated cycling produces necks of type $D$.

\section{B. Shape of the necks}

In order to determine the minimal cross section from the conductance using the modified Sharvin formula, Eq. (1), it is necessary to be certain that the necks are ballistic. For experiments performed at low temperature we have used PCS. Figure 6 shows the PCS curves corresponding to necks, that is steady-state plastic deformation cycles, similar to $D$, $C$, and $B$ in Fig. 1. For a given neck, the PCS curve does not change with the conductance [that is, the derivative of the conductance scales with $R^{3 / 2}$ as given by Eq. (2)], but the amplitudes of the phonon peaks are different for different necks. For a neck such as $D$, the amplitude of the phonon peaks is maximum (black curve in Fig. 6) and similar to that of previously reported spectra ${ }^{27}$ for ballistic point contacts, indicating that these necks are quite crystalline. ${ }^{25}$ In necks such as $B$ and $C$ the amplitude of the phonon peaks in the 


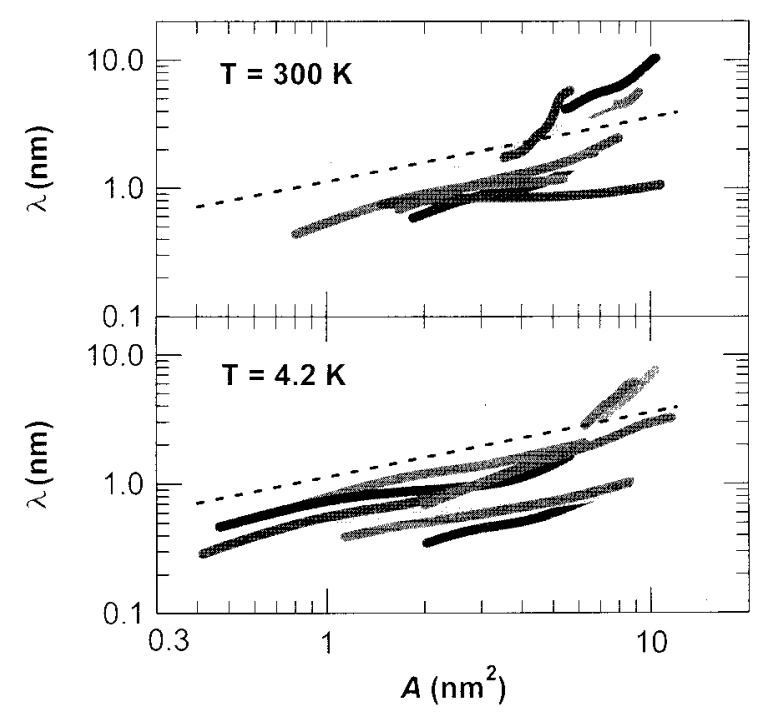

FIG. 7. Plastic deformation length vs minimal cross section of $\mathrm{Au}$ necks fabricated at room temperature and $4.2 \mathrm{~K}$. The dashed line represents $\lambda=2 \sqrt{A / \pi}$, i.e., an aspect ratio of one.

PCS curve is reduced (gray curves in Fig. 6) due to elastic scattering in the neck region, indicating the presence of defects, but still the necks are far from being disordered. From this evidence we conclude that our experimental metallic necks formed by plastic deformation are quite ballistic, and are not disordered. This idea is supported by the results of the molecular-dynamics simulations. ${ }^{11,12}$

In Fig. 1 we show four representative experimental sets of data. Each set consists of five elongation-contraction cycles. In order to use the previously defined slab model to deduce the shape of the corresponding necks, we have to find the equilibrium points (zero elastic force points) of each configuration, which is approximately in the middle of the hysteresis cycle, as illustrated in Fig. 5. The plastic deformation length $\lambda$ for these necks at the largest radius (approximately $1.9 \mathrm{~nm}$, corresponding to 120 conductance units) is, for curve $A, 0.2 \mathrm{~nm}$; for curve $B, 1 \mathrm{~nm}$; for curve $C, 2.8 \mathrm{~nm}$; and, for curve $D$, about $6 \mathrm{~nm}$. In all cases, except for curve $A$, this value decreases monotonically. Taking into account the physical meaning of $\lambda$, we can see that for neck D the amount of material involved in the plastic deformation is many times larger than for neck A. In this last case only one atomic layer is involved, while in the former case many layers redistribute in the process of plastic deformation.

In order to summarize all the different observed behaviors, in Fig. 7 we show a $\log -\log$ plot of $\lambda$ vs $A$ at room temperature and at $4.2 \mathrm{~K}$. The few curves plotted are representative of the observed behavior for hundreds of necks of cycles. All the observed curves are within the same region in the plot. The dashed line corresponds to a $\lambda$ equal to the diameter of the neck. Note that some curves lie above this line, that is, the longest necks are longer than their diameter. Naturally $\lambda$ decreases to a few tenths of a nanometer as the minimal section decreases to one corresponding to a few atoms. On the other hand, the shortest necks show small variations of $\lambda$, typically from 0.5 to $1 \mathrm{~nm}$. Necks formed at low and room temperature are not very different (at room temperature, long necks are somewhat easier to form and

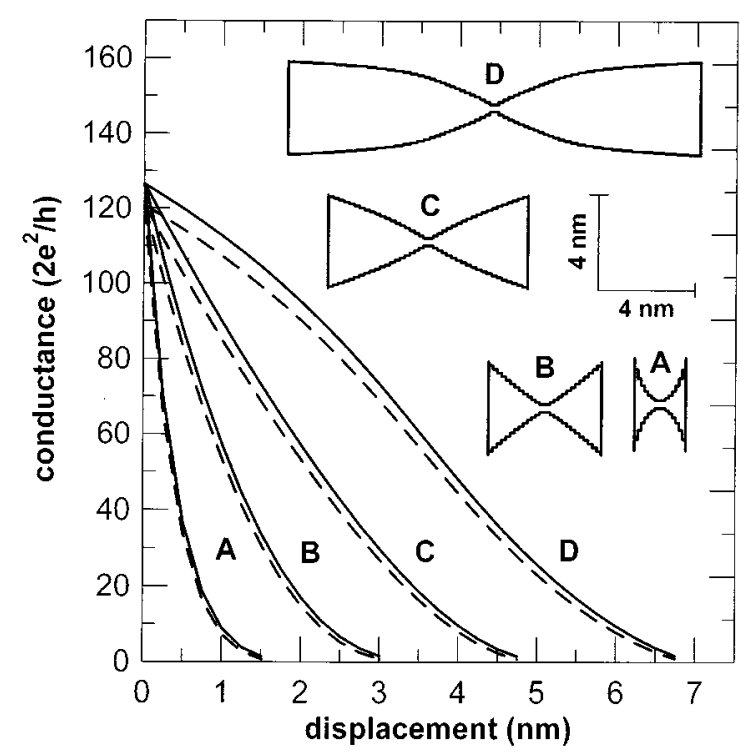

FIG. 8. Conductance and shapes of the necks in Fig. 1 using a slab model.

typically about $30 \%$ longer). This is to be expected, since room temperature is still much lower than the melting temperature for Au.

In Fig. 8 we show the geometry of four necks right before breaking. We have chosen the dependence of $\lambda$ on $A$ to be similar to that corresponding to the experimental necks in Fig. 1. This figure illustrates the fact that starting from the same cross-sectional area it is possible to have necks with very different geometries. We must emphasize that necks labeled $A$ and $D$ are the limiting cases. Neck $A$ (short neck) has a constant $\lambda \approx 0.2-0.3 \mathrm{~nm}$. On the other hand, neck $D$ (long neck or nanowire) corresponds to the longest observed necks. Note that according to the model the shape of the neck is only determined by $\lambda(A)$ and not by the initial cross sectional area of the neck. Neck $D$ not only is longer but also, as shown by the PCS curve, it has less defects, that is, it is more crystalline. Another interesting feature of these long necks is that their evolution is typically very repetitive. Figure 9 shows another long neck. The repetitiveness is remarkable, not only the five consecutive cycles superimpose almost perfectly, but the configurations appear to be the same (or very similar) for elongation and contraction. These long necks very often evolve repetitively for an indefinite number of cycles once the steady state is reached. This repetitiveness is not observed in short necks. This behavior is likely to be related to the deformation mechanism during plastic deformation. For short necks, relaxation would involve a structural transformation in which a portion of the neck of thickness $\lambda$, composed of just a few atomic layers, becomes disordered, and then rearranges to form an added layer, ${ }^{11,26}$ that is, some kind of order-disorder transition. This disorder precludes repetitiveness. On the other hand, the values of $\lambda$ for long necks indicate that the structural transformation involves many atomic layers. In this case the deformation mechanism is likely to involve gliding on (111) planes (as illustrated by the recent molecular-dynamics simulations in Refs. 13 and 28) instead of disorder and 


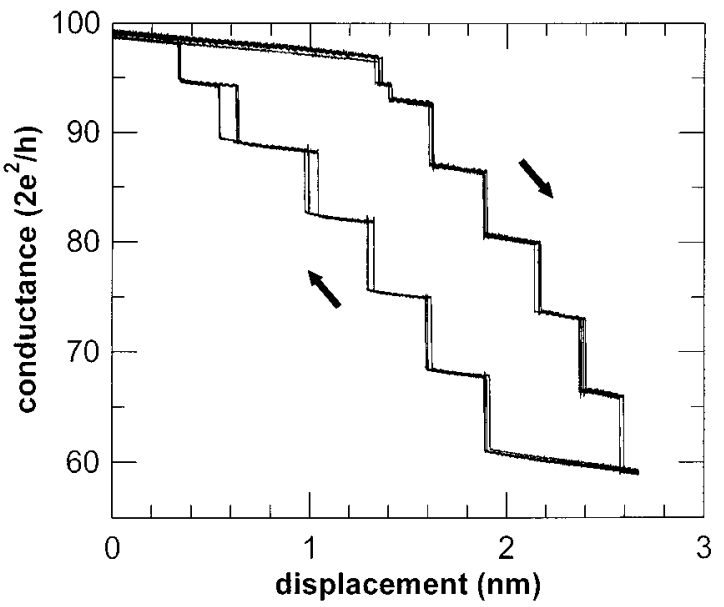

FIG. 9. Striking repetitiveness of the conductance during the cyclical plastic deformation of a long Au neck at $4.2 \mathrm{~K}$. This is a set of five consecutive elongation-contraction cycles. The neck goes through the same (or very similar) configurations during elongation and contraction in all the cycles.

rearrangement, ${ }^{11}$ making possible a cyclic evolution through almost identical configurations.

If the substrate is imaged right after breaking a neck, a protrusion is observed on the spot where the neck was formed. Figure 10 shows the profile of such a protrusion. The observed profile must be corrected (dashed line), taking into account that a similar protrusion is acting as a scanning tip. These dimensions are consistent with the shapes estimated using the slab model.

The geometries of the necks in Fig. 8 suggest that when a neck of type $A$ breaks at low temperatures, when surface difussion is negligible, the last few atoms should form a sharp spire. However, this kind of structure is unlikely to be stable due to surface tension. This final sharp structure retracts several tenths of nanometer, and only a blunt protrusion is visible.

The different types of necks described above depend on the history of the contact. In the case of Au illustrated here, short necks (type $A$ ) are typically obtained for the initial indentations, while long necks (type $D$ ) require a deeper indentation and repeated elongation and contraction. The fact that long necks present a prominent and repetitive stepped structure in the conductance (like the neck in Fig. 9), and a large amplitude of the phonon peaks in the PCS curve, indi-

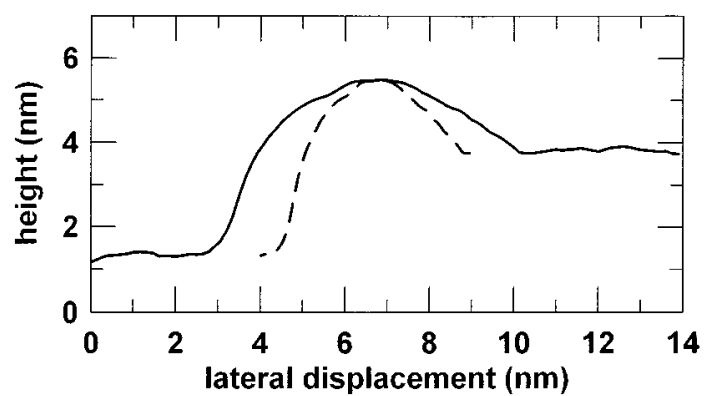

FIG. 10. Profile of a protrusion resulting after breaking a long $\mathrm{Au}$ neck $(T=4.2 \mathrm{~K})$. If we assume that the protrusion on the substrate is scanned with a similar protrusion acting as the STM tip the actual dimensions of the protrusion would be given approximately by the dashed line.

cates that the neck is quite crystalline. Possibly this repeated plastic deformation causes a "mechanical annealing" of the defects.

\section{CONCLUSIONS}

A simple slab model that correlates minimal crosssectional areas determined from the electrical conductance during the contraction and elongation process to the relative tip-substrate displacement can be used to estimate the shape of the metallic neck formed using a STM, and point-contact spectroscopy can be used to check that the constriction is indeed ballistic, which is essential for the correct determination of the minimal cross section.

Determination of the shape of experimental Au necks at low $(4.2 \mathrm{~K})$ and room temperatures shows that there is a strong dependence on the fabrication procedure, it being possible to form long necks (or nanowires) by repeated elongation and contraction, while necks formed at initial shallow indentations are typically short. The striking repetitiveness of the conductance during elongation-contraction cycles of the long necks, and the fact that electronic transport is ballistic, as indicated by PCS measurements, show that long necks are quite crystalline.

\section{ACKNOWLEDGMENT}

This work was supported by the DGICYT under Contract Nos. MAT95-1542 and PB94-0382.
${ }^{1}$ C. J. Muller, J. M. van Ruitenbeek, and L. J. de Jongh, Phys. Rev. Lett. 69, 140 (1992); J. M. Krans, J. C. Muller, I. K. Yanson, Th. C. M. Govaert, R. Hesper, and J. M. van Ruitenbeek, Phys. Rev. B 48, 14721 (1993); N. van der Post, E. T. Peters, I. K. Yanson, and J. M. van Ruitenbeek, Phys. Rev. Lett. 73, 2611 (1994); J. M. Krans and J. M. van Ruitenbeek, Phys. Rev. B 50, 17659 (1994); J. M. Krans, J. M. van Ruitenbeek, V. V. Fisun, I. K. Yanson, and L. J. de Jongh, Nature (London) 375, 767 (1995).

${ }^{2}$ N. Agraï, J. G. Rodrigo, and S. Vieira, Phys. Rev. B 47, 12345
(1993); N. Agrait, J. G. Rodrigo, C. Sirvent, and S. Vieira, ibid. 48, 8499 (1993).

${ }^{3}$ J. G. Rodrigo, N. Agraï, and S. Vieira, Phys. Rev. B 50, 374 (1994).

${ }^{4}$ J. G. Rodrigo, N. Agraï, C. Sirvent, and S. Vieira, Phys. Rev. B 50, 7177 (1994); 50, 12788 (1994).

${ }^{5}$ D. P. E. Smith, Science 269, 371 (1995).

${ }^{6}$ J. I. Pascual, J. Méndez, J. Gómez-Herrero, A. M. Baró, N. García, and Vu Thien Binh, Phys. Rev. Lett. 71, 1852 (1993); J. I. 
Pascual, J. Méndez, J. Gómez-Herrero, A. M. Baró, N. García, U. Landman, W. D. Luedtke, E. N. Bogachek, and H. -P. Cheng, Science 267, 1793 (1995).

${ }^{7}$ L. Olesen, E. Lægsgaard, I. Stensgaard, F. Besenbacher, J. Schiøtz, P. Stolze, K. W. Jacobsen, and J. K. Nørskov, Phys. Rev. Lett. 72, 2251 (1994).

${ }^{8}$ N. Agraït, J. G. Rodrigo, G. Rubio, C. Sirvent, and S. Vieira, Thin Solid Films 253, 199 (1994); N. Agraï, G. Rubio, and S. Vieira, Langmuir 12, 4505 (1996); N. Agraï, G. Rubio, and S. Vieira, Phys. Rev. Lett. 74, 3995 (1995).

${ }^{9}$ A. Stalder and U. Dürig, Appl. Phys. Lett. 68, 637 (1996); J. Vac. Sci. Technol. B 14, 1259 (1996).

${ }^{10}$ G. Rubio, N. Agraï, and S. Vieira, Phys. Rev. Lett. 76, 2302 (1996).

${ }^{11}$ U. Landman, W. D. Luedtke, N. A. Burnham, and R. J. Colton, Science 248, 454 (1990).

${ }^{12}$ R. M. Lynden-Bell, J. Phys., Condens. Matter. 4, 2127 (1992); Science 263, 1704 (1994).

${ }^{13}$ U. Landman, W. D. Luedtke, B. E. Salisbury, and R. L. Whetten, Phys. Rev. Lett. 77, 1362 (1996).

${ }^{14}$ T. N. Todorov and A. P. Sutton, Phys. Rev. Lett. 70, 2138 (1993); A. M. Bratkovsky, A. P. Sutton, and T. N. Todorov, Phys. Rev. B 52, 5036 (1995).

${ }^{15}$ E. N. Bogachek, A. M. Zagoskin, and I. O. Kulik, Fiz. Nizk. Temp. 16, 1404 (1990) [Sov. J. Low Temp. Phys. 16, 796 (1990)].
${ }^{16}$ J. A. Torres, J. I. Pascual, and J. J. Sáenz, Phys. Rev. B 49, 16 581 (1994).

${ }^{17}$ J. A. Torres and J. J. Sáenz, Physica B 218, 234 (1996).

${ }^{18}$ A. M. Bratkovsky and S. N. Rashkeev, Phys. Rev. B 53, xxx (1996).

${ }^{19}$ J. K. Gimzewski and R. Möller, Phys. Rev. B 36, 1284 (1987).

${ }^{20}$ The mean free path of the electrons in $\mathrm{Au}$ is about $30 \mathrm{~nm}$ at room temperature and much larger at low temperature.

${ }^{21}$ Yu. V. Sharvin, Zh. Eksp. Teor. Fiz. 48, 984 (1965) [Sov. Phys. JETP 21, 655 (1965)].

${ }^{22}$ A. G. M. Jansen, A. P. van Gelder, and P. Wyder, J. Phys. C 13, 6073 (1980).

${ }^{23}$ A. García-Martín, J. A. Torres, and J. J. Sáenz, Phys. Rev. B 54, 13448 (1996).

${ }^{24}$ P. García-Mochales, P. A. Serena, N. García, and J. L. CostaKrämer, Phys. Rev. B 53, 10268 (1996).

${ }^{25}$ I. K. Yanson, Fiz. Nizk. Temp. 9, 676 (1983) [Sov. J. Low Temp. Phys. 9, 343 (1983)]; A. M. Duif, A. G. M. Jansen, and P. Wyder, J. Phys., Condens. Matter. 1, 3157 (1989); see also D. C. Ralph and R. A. Buhrman, Phys. Rev. B 51, 3554 (1995).

${ }^{26}$ J. A. Torres and J. J. Saénz, Phys. Rev. Lett. 77, 2245 (1996).

${ }^{27}$ A. G. M. Jansen, F. M. Mueller, and P. Wyder, Phys. Rev. B 16, 1325 (1977).

${ }^{28}$ P. Español, I. Zuñiga, G. Rubio, and N. Agraï (unpublished). 